\title{
Second harmonic generation in nonlinear disordered media
}

\author{
Wieslaw Krolikowski \\ Laser Physics Centre, Research School of Physical Sciences and Engineering, \\ Australian National University, Canberra, Australia
}

The efficient energy transfer in nonlinear parametric optical processes such as second harmonic generation ( $\mathrm{SHG}$ ) require phase-matching between the interacting waves. In order to fulfill this condition various methods have been developed, including the quasiphase matching (QPM) technique which relies on the spatially periodic modulation of the medium's nonlinear properties [1]. In case of ferroelectric crystals this is typically achieved by inducing periodic anti-parallel ferroelectric domain structure which leads to periodic reversal of the sign of quadratic nonlinearity. However, as the modulation period critically depends on the wavelengths of interacting waves, once formed structure is only effective for the particular choice of the wavelength and the nonlinear process, hence limiting its practical applicability. To overcome this restriction and broaden the useful range of the wavelengths one needs to fabricate costly multi-period or chirped structures.

It has been recently demonstrated that a broadband SHG can be achieved in ferroelectric crystal with random distribution of ferroelectric domains [2]. In such crystal the second harmonic wave can be generated in a form of a plane [see Fig.1(b)] or cone depending on the direction of the fundamental beam with respect to the orientation of anti-parallel domains. We used strontium barium niobate crystal (SBN) to demonstrate SHG with femtosecond pulses. This process relies on the fact, that naturally grown ferroelectric crystals exhibit multi-domain structure with domains having random distribution of size and orientation [see Fig 1(a)]. Such a disordered nonlinear medium [3] is equivalent to an effective QPM system with almost infinite set of reciprocal wave vectors enabling to quasi-phase-match any parametric process, e.g. SHG or sum-frequency mixing in an ultra-broad frequency range which is limited only by transparency window of the material. In case of SBN crystals the anti-parallel domains are tens-of-microns-long with a typical diameter from one to few microns [4].

The ability to achieve broadband SHG can find an application in simple auto-correlator which can be used in short pulse diagnostics. We demonstrate this concept by realizing simple auto-correlator with counter-propagating femtosecond pulses [5] shown in Fig.1 (c). When pulses propagate in the direction perpendicular to the domains the secondharmonic signal consists of two components - first, generated by each individual pulse, and second, created by mixing of photons from both pulses. The latter is the autocorrelation signal while the former forms a background. Fig.1(d-e) depicts typical autocorrelation traces as seen with the CCD camera for pulses with tilted (d) pulse front and for sequence of two closely separated pulses (e). The sensitivity of this simple device can be greatly enhanced by propagating both pulses along the optical axis which coincides with the domains long dimension. In this case the transversely mounted CCD 
camera will record the second-harmonic signal generated only by counter-propagating pulses in their overlapping region leading to a background-free autocorrelation trace [see Fig. 1(f)].

(a)

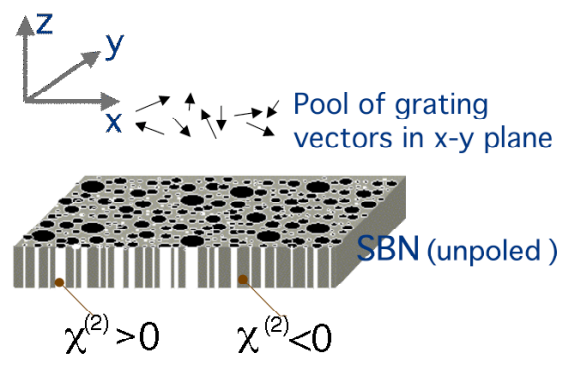

(d)

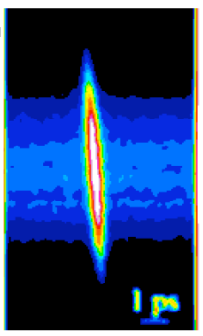

(b)

(e)
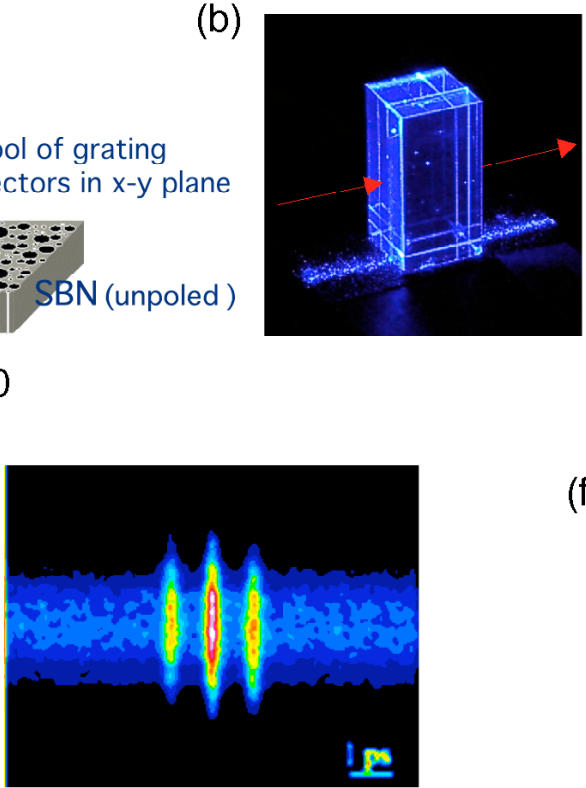

(c)

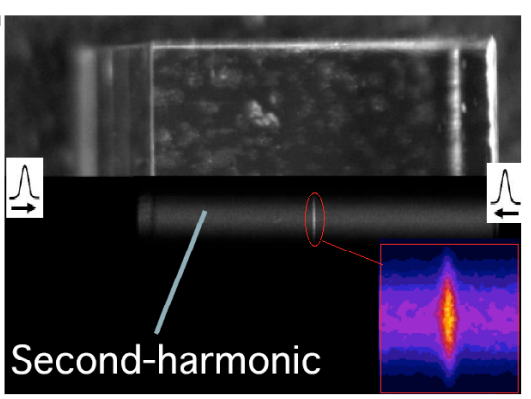

(f)

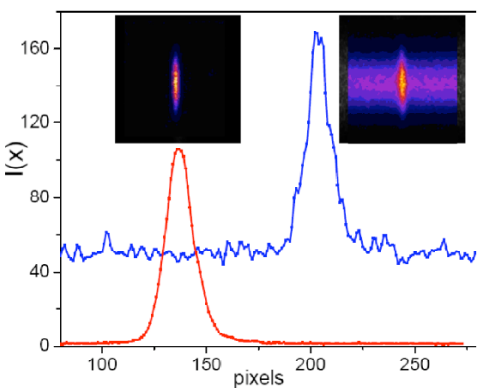

Figure 1. (a) Schematic representation of random domain distribution in SBN crystal; (b) photo of planar emission of second harmonic (arrows shows propagation direction of the fundamental wave); (c) illustrating the concept of the pulse autocorrelator in disordered crystal - inset shows detail of the auto-correlation signal; (d-e) autocorrelation signals corresponding to pulse with the tilted pulse front (d) and sequence of two separated pulses (e); (f) autocorrelation trace for pulses propagating perpendicularly (blue line visible background) and along the ferroelectric domains (red line - background free geometry).

\section{References:}

1. M.M. Fejer, G.A. Magel, D.H. Jundt, and R.L. Byer, IEEE J. Quant. Electron. QE28, 2631 (1992).

2. A.R. Tunyagi, M. Ulex, and K. Betzler, Phys. Rev. Lett. 90, 243901 (2003).

3. R. Fischer, S. M. Saltiel, D. N. Neshev, W. Krolikowski, and Yu.S. Kivshar, Appl. Phys. Lett. 89, 191105 (2006).

4. M. Baudrier-Raybaut, R. Haidar, Ph. Kupecek, Ph. Lemasson, and E. Rosencher, Nature 432, 374 (2004); J.J. Romero, C. Arago, J.A. Gonzalo, D. Jaque, and J. Garcia Sole, J. Appl. Phys. 93, 3111 (2003).

5. R. Fischer, S. M. Saltiel, D. N. Neshev, A. Sukhorukov, W. Krolikowski, and Yu.S. Kivshar, Appl. Phys. Lett. 91, 031104 (2007); V. Roppo, C. Cojocaru, R. Fischer, S. Saltiel, K. Staliunas, R. Herrero, R. Vilaseca, D. Neshev, W. Krolikowski, Yu. Kivshar, Opt. Express 16, 14192 (2008) 